\title{
Long-term Outcomes of Carbon-ion Radiotherapy for Locally Advanced Squamous Cell Carcinoma of the Uterine Cervix
}

\author{
NORIYUKI OKONOGI ${ }^{1}$, MASARU WAKATSUKI ${ }^{2}$, SHINGO KATO $^{3}$, SHINTARO SHIBA ${ }^{4}$, DAIJIRO KOBAYASHI ${ }^{4}$, \\ HIROKI KIYOHARA ${ }^{5}$, KUMIKO KARASAWA ${ }^{6}$, TATSUYA OHNO ${ }^{4}$, TAKASHI NAKANO ${ }^{4}$, \\ TADASHI KAMADA ${ }^{1}$, MAKIO SHOZU ${ }^{7}$ and THE WORKING GROUP OF GYNECOLOGICAL TUMORS \\ ${ }^{1}$ Hospital, National Institute of Radiological Sciences, \\ National Institutes for Quantum and Radiological Science and Technology, Chiba, Japan; \\ ${ }^{2}$ Department of Radiology, Jichi Medical University, Tochigi, Japan; \\ ${ }^{3}$ Department of Radiation Oncology, Saitama Medical University, International Medical Center, Saitama, Japan; \\ ${ }^{4}$ Department of Radiation Oncology, Gunma University Graduate School of Medicine, Gunma, Japan; \\ ${ }^{5}$ Department of Radiation Oncology, Maebashi Red Cross Hospital, Maebashi, Japan; \\ ${ }^{6}$ Department of Radiation Oncology, Tokyo Women's Medical University, Tokyo, Japan; \\ ${ }^{7}$ Department of Reproductive Medicine, Graduate School of Medicine, Chiba University, Chiba, Japan
}

\begin{abstract}
Background/Aim: To evaluate the long-term outcomes of carbon-ion radiotherapy (C-ion $R T$ ) for locally advanced cervical squamous cell carcinoma (LAC-SqCC) in 2 prospective phase I/II studies. Patients and Methods: In the first study, 14 patients were treated with 68.8-72.8 Gy (RBE)/24 fractions. In a subsequent study, 22 patients were treated with 64.0-72.0 Gy (RBE)/20 fractions while limiting the dose to the gastro-intestinal $(G I)<60$ Gy (RBE). Results: The 10-year local control rates were $92 \%$ and $61 \%$ for the patients administered a total dose of 72.0-72.8 Gy (RBE) and 64.0-68.8 Gy (RBE), respectively. Two patients in the first study developed grade $3 / 4$ rectal or bladder complications; however, no grade 3 or higher complications occurred in the second study. Conclusion: $C$-ion $R T$ with a higher dose was associated with excellent $L C$ in LAC-SqCC. Long-term safety was confirmed based on the establishment of dose constraints for the GI tract.
\end{abstract}

Uterine cervical cancer is the third most common cancer affecting women globally (1). Several randomized phase III

This article is freely accessible online.

Correspondence to: Dr. Noriyuki Okonogi, Hospital, National Institute of Radiological Sciences, National Institutes for Quantum and Radiological Science and Technology, 4-9-1 Anagawa, Inageku, Chiba 263-8555, Japan. Tel: +81 432063306, Fax: +81 432566506, e-mail: okonogi.noriyuki@qst.go.jp

Key Words: Carbon-ion radiotherapy, particle radiation therapy, radiotherapy, uterine cervical cancer, squamous cell carcinoma. clinical trials and meta-analyses related to this disease demonstrated that concurrent chemoradiotherapy (CCRT) improved local control (LC) and overall survival (OS) when compared to radiotherapy alone (2-4). Intracavitary brachytherapy plays an important role in the treatment of patients with uterine cervical cancer. However, conventional intracavitary brachytherapy with tandem and ovoid systems might not deliver a sufficient dose in the case of extensive and bulky tumors, resulting in unfavorable LC for patients with Stage III or IVA disease (5-8). Interstitial brachytherapy may be one of the treatment options for such tumors $(9,10)$; however, it is invasive, technically difficult, and has limited indications.

In 1994, clinical studies of carbon-ion radiotherapy (C-ion RT) were initiated at the National Institute of Radiological Sciences of Japan (NIRS) (11). Carbon-ion beams possess improved dose localization properties, which can potentially enhance anti-tumor effects markedly while minimizing normal tissue damage $(12,13)$. Moreover, they possess a biological advantage due to their high linear energy transfer in the Bragg Peak (14). Several phase I/II or phase II clinical trials investigating $\mathrm{C}$-ion RT in various types of malignant tumors have been conducted at the NIRS; these studies aimed to shorten treatment fractions and times of exposure while being less invasive than the standard therapy (11). Although concurrent chemotherapy with external RT and intracavitary brachytherapy is considered the standard therapy for locally advanced uterine cervical cancer, the C-ion RT clinical trials conducted at the NIRS aimed to improve LC and survival outcomes while minimizing late radiation toxicities, especially for locally advanced bulky 
cervical cancer. During this period, we reported the results of several prospective clinical trials using C-ion RT for patients with cervical cancer (15-18). To the best of our knowledge, studies aimed at investigating the long-term safety and efficacy of C-ion RT have yet to be performed. Hence, we conducted a long-term follow-up study of C-ion RT for locally advanced squamous cell carcinoma (SqCC) of the uterine cervix.

\section{Patients and Methods}

Patient eligibility. This study included all patients enrolled in protocols 9702 and 9902, which were 2 dose-escalation studies aimed at assessing the feasibility of C-ion RT for locally advanced $\mathrm{SqCC}$ of the uterine cervix. Fourteen patients were enrolled in the protocol 9702 study, which was performed between December 1997 and January 2000. Afterwards, 22 patients were enrolled in the protocol 9902 study, which was performed between April 2000 and January 2006. Hence, a total of 36 patients were included in the present study.

The eligibility criteria for the protocols were: (i) previously untreated, (ii) histologically proven $\mathrm{SqCC}$ of the uterine cervix, (iii) International Federation of Gynecology and Obstetrics (FIGO) stage IIB, III, or IVA disease, and (iv) tumor size $>4 \mathrm{~cm}$ in diameter. Other eligibility criteria included World Health Organization performance status $<3$, age $<80$ years, and estimated life expectancy $\geq 6$ months. Patients were excluded if they had a severe pelvic infection, severe psychological illness, or an active comorbid cancer. All patients underwent computed tomography (CT) of the abdomen and pelvis as well as magnetic resonance imaging (MRI) of the pelvis. Patients were staged according to the FIGO system, and those with para-aortic lymph nodes $\geq 1 \mathrm{~cm}$ in minimum diameter on $\mathrm{CT}$ images were excluded. However, patients with only enlarged pelvic lymph nodes were included in the studies. Tumor size was assessed by both pelvic examination and MRI; T2-weighted images were used to measure the dimensions of the cervical tumors. The working group pathologists reviewed the tumor specimens; other pretreatment evaluation included the assessment of patient history, physical and pelvic examination by gynecologists and radiation oncologists, routine blood cell counts, chemistry profiles, chest radiography, cystoscopy, and rectoscopy. Bladder or rectal involvement was assessed endoscopically.

The treatment protocols were reviewed and approved by the Ethics Committee of Human Clinical Research, and all patients provided their written informed consent to participate in the studies before the initiation of therapy.

Treatment planning of C-ion RT. A set of 5-mm-thick CT images was acquired for treatment planning. Three-dimensional treatment planning was performed using the HIPLAN software (National Institute of Radiological Sciences, Chiba, Japan) for C-ion RT planning (19). The treatment in both study protocols consisted of whole pelvic and local boost irradiation. The radiation dose was calculated for both the target volume and surrounding normal structures, and was expressed in Gy relative biological effectiveness (RBE), which is defined as the physical dose multiplied by the relative biologic effectiveness of the carbon ions $(12,13,19)$. The gross tumor volume (GTV) and the clinical target volume (CTV) were defined by CT and MRI as well as clinical examination immediately before each treatment planning session.
The GTV was defined as a high-intensity region on T2-weighted MRI. The CTV-1 was defined as the primary lesion (the GTV, enlarged lymph nodes with a 5-mm margin, uterus, bilateral parametrium, ovaries, and at least the upper half of the vagina) as well as the whole pelvic lymph node region (common iliac, internal iliac, external iliac, and obturator and presacral node regions). The CTV-2 was defined as the primary lesion and the enlarged lymph nodes. The planning target volume (PTV-1) included the CTV-1 plus a 5-mm margin for positioning uncertainty. Additionally, the uterus plus a $15-\mathrm{mm}$ margin for intra- and inter-fractional organ movement was included in the PTV-1. A 5 to $10-\mathrm{mm}$ margin was added to the CTV-2 to create the PTV-2.

In protocol 9702, the PTV-1 was covered with at least $90 \%$ of the prescribed dose, which was fixed at $44.8 \mathrm{~Gy}$ (RBE) in 16 fractions. After the completion of irradiation to PTV-1, a dose of 24.0 or $28.0 \mathrm{~Gy}(\mathrm{RBE})$ in 8 fractions to the PTV-2 was delivered in a dose-escalating manner. The total dose to the GTV was 68.8 or 72.8 Gy (RBE) in 20 fractions.

In protocol 9902, the PTV-1 and PTV-2 were the same as defined in protocol 9702, and a dose of $39.0 \mathrm{~Gy}$ (RBE) in 13 fractions was delivered to the PTV-1. With respect to local boost irradiation, a fixed dose of $15.0 \mathrm{~Gy}$ (RBE) was delivered in 5 fractions. Next, the CTV was further shrunk to just the GTV (CTV-3), with a 3-mm margin added, to create the PTV-3. Normal tissues such as the rectum, sigmoid colon, bladder, and the small bowel in the pelvis were completely excluded from the PTV-3. A dose-escalation was planned with doses of $10.0,14.0$, or $18.0 \mathrm{~Gy}$ (RBE) in 2 fractions to the PTV-3. The total dose to the GTV in protocol 9902 was 64.0$72.0 \mathrm{~Gy}$ (RBE) in 20 fractions. The dose constraints were as follows: rectum and sigmoid colon, $<60 \mathrm{~Gy}$ (RBE); small intestine, $<54$ Gy (RBE); and bladder, <70 Gy (RBE) maximum with the V40 Gy (RBE) (percentage of the bladder volume receiving at least 40 Gy (RBE)) at $\leq 33 \%$ to prevent major late toxicities.

To minimize the internal motion of the uterine cervix, 100-150 $\mathrm{ml}$ of normal saline was infused into the bladder, and vaginal packing was performed tightly at each treatment session. Moreover, the cotton used for vaginal packing was soaked in a contrast medium so that the surface of the uterine cervix could be visualized by radiography during the treatment sessions.

Treatment modality of C-ion RT. The Heavy Ion Medical Accelerator in Chiba (HIMAC) is the world's first heavy ion accelerator complex dedicated to medical use in a hospital environment. The $\mathrm{C}$-ion RT treatment system was described previously $(12,13)$. Carbon ion beams are generated by the HIMAC; the accelerated beam energies are 290, 350, 400, and 430 $\mathrm{MeV}$. The beam range achieves a depth of $15-25 \mathrm{~cm}$ in water. An appropriately sized ridge filter corresponding to the tumor size is selected to form the spread-out Bragg peak. The compensation bolus is fabricated for each patient in a manner where the distal configuration of the spread-out Bragg peak is similar to the shape of the target volume. A multileaf or patient collimator, adapted to the radiation field for each target volume, defines the margins of the target volume.

Patients were positioned in customized cradles and immobilized with a low-temperature thermoplastic sheet. Patients received C-ion RT 4 consecutive days per week. At every treatment session, a patient's position was verified with respect to bone structure with a computer-aided, on-line positioning system. Digital orthogonal radiographic images were acquired and transferred to the 
Table I. Patient characteristics.

\begin{tabular}{|c|c|c|c|c|}
\hline \multirow[t]{2}{*}{ Characteristics } & \multirow{2}{*}{$\begin{array}{c}\text { Protocol } 9702 \\
\text { Number }\end{array}$} & \multirow{2}{*}{$\begin{array}{c}\text { Protocol } 9902 \\
\text { Number }\end{array}$} & \multicolumn{2}{|c|}{ Total } \\
\hline & & & Number & $(\%)$ \\
\hline Number of patients & 14 & 22 & 36 & \\
\hline $\begin{array}{l}\text { Median age, years old } \\
\text { (range) }\end{array}$ & $\begin{array}{c}61 \\
(50-79)\end{array}$ & $\begin{array}{c}57 \\
(31-80)\end{array}$ & $\begin{array}{c}57 \\
(31-80)\end{array}$ & \\
\hline \multicolumn{5}{|l|}{ FIGO stage } \\
\hline IIB & 0 & 1 & 1 & (3) \\
\hline III & 9 & 18 & 27 & (75) \\
\hline IVA & 5 & 3 & 8 & (22) \\
\hline \multicolumn{5}{|l|}{$\begin{array}{l}\text { Tumor size in } \\
\text { maximum diameter }\end{array}$} \\
\hline$\leq 6 \mathrm{~cm}$ & 4 & 12 & 16 & (44) \\
\hline$>6 \mathrm{~cm}$ & 10 & 10 & 20 & (56) \\
\hline \multicolumn{5}{|l|}{ Pelvic LN metastasis } \\
\hline Yes & 11 & 7 & 18 & $(50)$ \\
\hline No & 3 & 15 & 18 & (50) \\
\hline $\begin{array}{l}\text { Median OTT, days } \\
\text { (range) }\end{array}$ & $\begin{array}{c}42 \\
(35-48)\end{array}$ & $\begin{array}{c}34 \\
(32-37)\end{array}$ & $\begin{array}{c}36 \\
(32-48)\end{array}$ & \\
\hline \multicolumn{5}{|l|}{ Total dose } \\
\hline 64.0 Gy (RBE) & - & 11 & 11 & (31) \\
\hline 68.0 Gy (RBE) & - & 5 & 5 & (14) \\
\hline 68.8 Gy (RBE) & 7 & - & 7 & (19) \\
\hline $72.0 \mathrm{~Gy}$ (RBE) & - & 6 & 6 & (17) \\
\hline 72.8 Gy (RBE) & 7 & - & 7 & (19) \\
\hline
\end{tabular}

FIGO: International Federation of Gynecology and Obstetrics; LN: lymph node; OTT: overall treatment time.

positioning computer. The positioning images were compared to the reference images that were digitally reconstructed from the CT scans. If the difference in positioning was $>2 \mathrm{~mm}$, the treatment couch was moved until an acceptable position was attained.

Assessment of efficacy and toxicity. After completing treatment, patients received follow-up every 1-3 months for 2 years, and every 3 or 6 months thereafter. The effect of the treatment was evaluated in terms of LC, disease-free survival (DFS) and OS. LC and DFS were defined as showing no evidence of tumor regrowth or recurrence in the treatment volume, respectively, on physical examination, CT, MRI, positron emission tomography, and/or biopsy. LC, DFS, and OS rates were calculated by using the KaplanMeier method, and the log-rank test was used for comparisons. Late toxicity was graded according to the Radiation Therapy Oncology Group/European Organization for Research and Treatment of Cancer late radiation morbidity scoring scheme (20). All statistical analyses were performed using SPSS 16.0 for Mac (SPSS, Chicago, IL, USA).

\section{Results}

The median age of the patients was 57 years (range $=31-80$ years). One patient had stage IIB, 27 had stage IIIB, and 8 had stage IVA disease. The median tumor size was $6.5 \mathrm{~cm}$ (range $=4.2-12.0 \mathrm{~cm}$ ). Eighteen of the 36 patients had pelvic
Table II. Late maximal toxicities by RTOG/EORTC scoring.

\begin{tabular}{|c|c|c|c|c|c|c|c|}
\hline \multirow[b]{2}{*}{ Protocol } & \multirow[b]{2}{*}{ Site } & \multirow[b]{2}{*}{$\begin{array}{l}\text { No. of } \\
\text { patients }\end{array}$} & \multicolumn{5}{|c|}{ RTOG/EORTC Grade } \\
\hline & & & 0 & 1 & 2 & 3 & 4 \\
\hline \multirow[t]{4}{*}{9702} & Rectum & 14 & 5 & 5 & 2 & $1^{\mathrm{a}}$ & $1^{\mathrm{b}}$ \\
\hline & Bladder & 14 & 11 & 0 & $2^{\mathrm{a}}$ & 0 & $1^{\mathrm{b}}$ \\
\hline & Small intestine & 14 & 13 & 0 & $1^{\mathrm{a}}$ & 0 & 0 \\
\hline & Bone & 14 & 13 & 0 & 1 & 0 & 0 \\
\hline \multirow[t]{4}{*}{9902} & Rectum & 22 & 14 & 7 & 1 & 0 & 0 \\
\hline & Bladder & 22 & 14 & 6 & 2 & 0 & 0 \\
\hline & Small intestine & 22 & 21 & 1 & 0 & 0 & 0 \\
\hline & Bone & 22 & 22 & 0 & 0 & 0 & 0 \\
\hline
\end{tabular}

a,bPatients who developed toxicities in multiple sites. RTOG/EORTC: Radiation therapy oncology group/European organization for research and treatment of cancer; No.: number.

lymph node metastases. The median follow-up periods were 36 months (range $=9-215$ months) for all patients, and 183 months (range $=121-215$ months) for surviving patients (Table I).

Antitumor effect. Ten patients developed local recurrences, all of which occurred within 2 years after treatment. At the most recent follow-up date, 23 patients died (18 patients of cervical cancer, 2 of other malignancies, and 3 of nonmalignant diseases).

The LC, DFS, and OS rates at 5 and 10 years after treatment were $72 \%, 47 \%$, and $47 \%$; and $72 \%, 42 \%$, and $39 \%$; respectively (Figure 1). The 10-year LC rates in patients with Stage II, III, and IVA were $100 \%, 74 \%$, and $63 \%$, respectively (Figure 2 ). The LC rates in patients with tumors $>6 \mathrm{~cm}$ versus those with tumors $\leq 6 \mathrm{~cm}$ were not significantly different (Figure 3). The 10-year LC rates in patients who received 72.0-72.8 Gy (RBE) (13 cases) were significantly better than in those who received 64.0-68.8 Gy (RBE) ( $p=0.043)$ (23 cases) (Figure 4$)$.

Late toxicity. Grade 2 or higher late toxicity occurred in 9 patients, with some patients experiencing toxicities at multiple sites (Table II). One patient developed grade 2 bone toxicity in the ileum and another developed grade 2 small bowel toxicity. Almost all of these toxicities were observed within 3 years after treatment, although the grade 2 toxicity in the small intestine was observed 10 years after treatment. Two patients had grade $3 / 4$ rectal and/or bladder complications; one had grade 4 rectal and bladder complications, the other had grade 3 rectal complications. Both patients required surgery.

Moreover, both the patients who experienced grade $3 / 4$ complications had been treated with a total dose of $72.8 \mathrm{~Gy}$ 


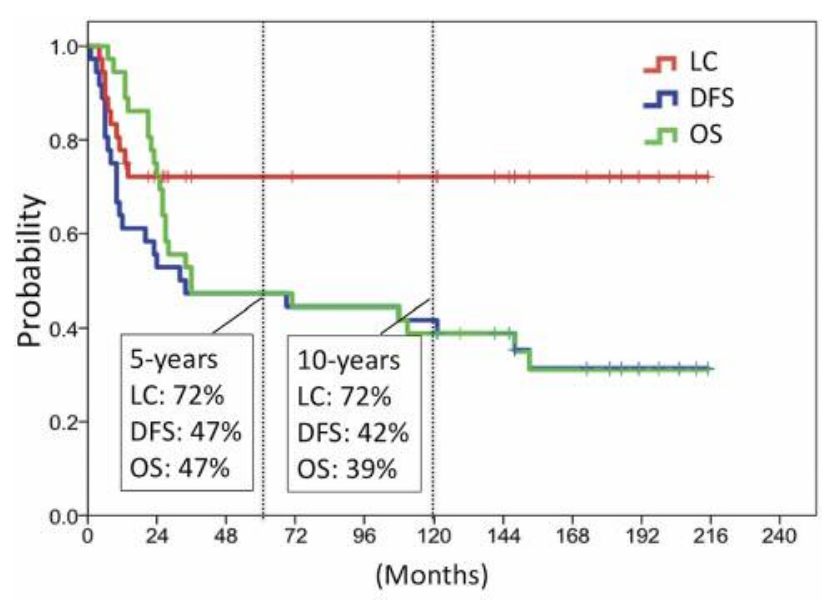

Figure 1. The local control, pelvic disease-free survival, and overall survival rates in all patients.

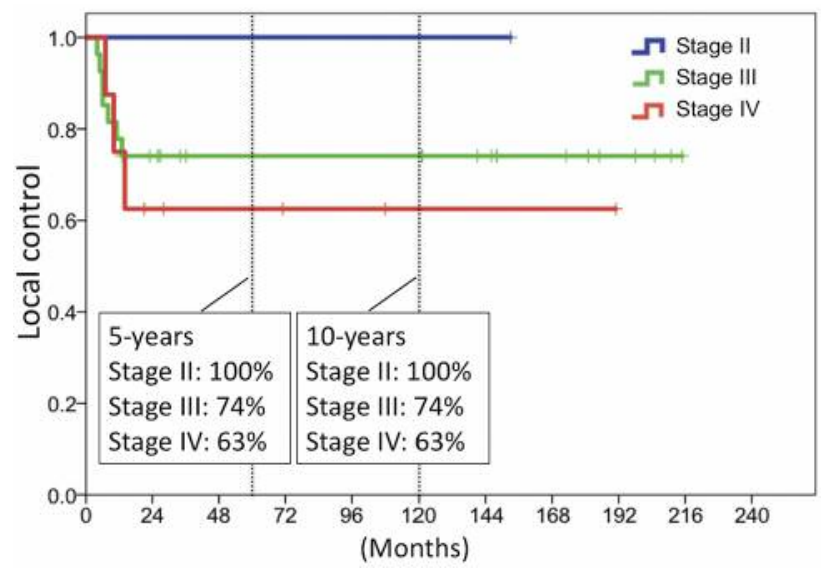

Figure 2. The local control rates classified by the International Federation of Gynecology and Obstetrics (FIGO) stage.

(RBE) in protocol 9702; the maximal doses to the rectum in these patients were 67.0 and $68.2 \mathrm{~Gy}$ (RBE), respectively. No grade 3 or higher late complications were observed among protocol 9902 patients.

\section{Discussion}

To the best of our knowledge, ours is the first study to show the long-term safety and efficacy of C-ion RT administration to the whole pelvis to treat locally advanced cervical cancer. The patients in the study were treated with $\mathrm{C}$-ion RT alone without chemotherapy because the safety of combination CCRT and C-ion RT had not been thoroughly evaluated at the time of the study. Even though

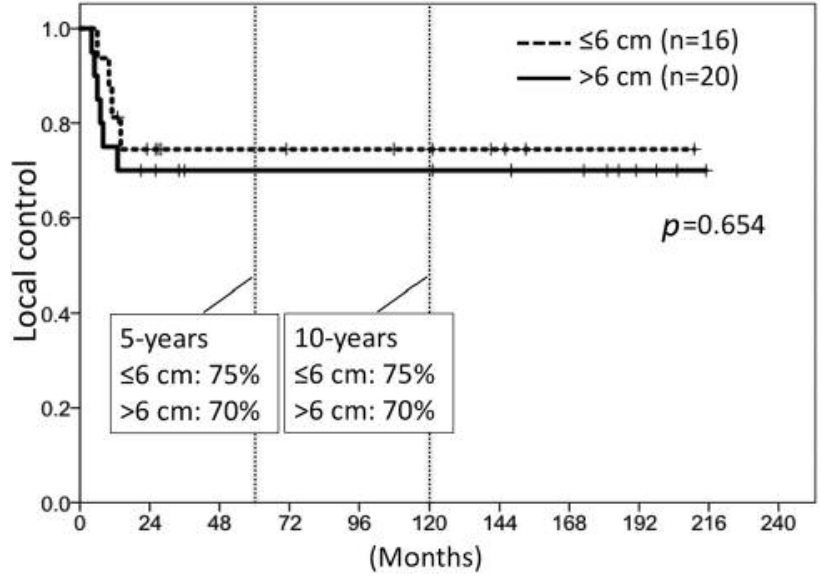

Figure 3. The local control rates classified by tumor size.

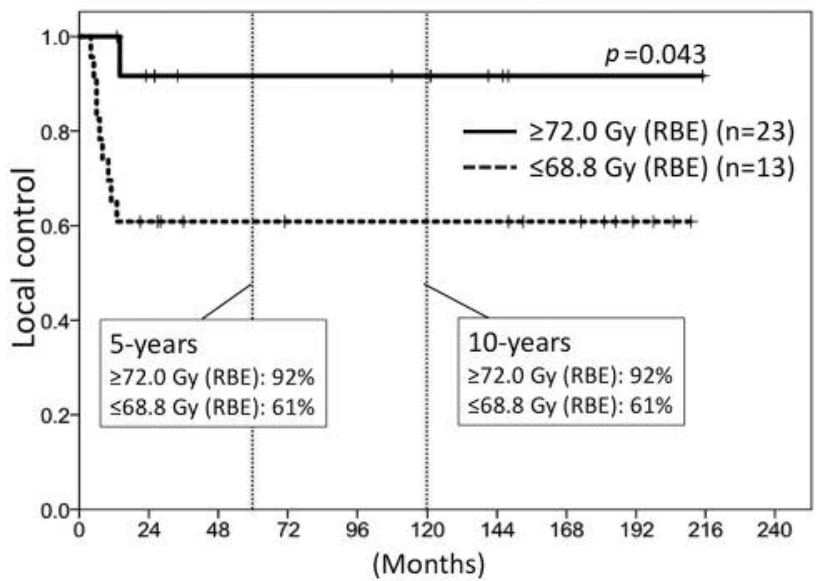

Figure 4. The local control rates classified by total carbon-ion radiotherapy dose.

our cohort included patients with extensive and bulky tumors, the 10-year LC rate in patients who received 72.072.8 Gy (RBE) was excellent (Figure 4). Furthermore, no protocol 9902 patient developed severe late toxicity (Table II). These results suggest that C-ion RT produces favorable LC with minimal radiation toxicity for locally advanced cervical cancer.

Tumor size is one of the significant prognostic factors in RT or CCRT for cervical cancer. Toita et al. reported a decrease in LC and survival in patients with larger tumor diameters following conventional CCRT (21). On the other hand, the LC rate of patients who had tumors $>6 \mathrm{~cm}$ was not statistically inferior to that of patients with larger tumors in the present study (Figure 3). This may be explained by 
the high-dose localization properties of $\mathrm{C}$-ion beams aimed at any irregularly shaped target. Recent studies have shown 3-dimensional (3D) image-guided brachytherapy (IGBT) for cervical cancer to produce favorable LC rates (22-26). Nowadays, 3D-IGBT is a critical component of definitive RT in patients with cervical cancer. However, in the case of extensive, bulky cervical cancer, it is difficult to deliver a sufficient dose to the whole tumor volume with conventional intracavitary brachytherapy (27). Fokdal et al. demonstrated that interstitial brachytherapy, not intracavitary brachytherapy, was required to achieve a favorable LC rate for bulky cervical cancer (27). Generally, interstitial brachytherapy is rather invasive, and can sometimes be technically difficult because of anatomic limitations. Taken together, C-ion RT may be a less invasive treatment option for the locally advanced cervical cancer, and can deliver an appropriately high dose to the large, irregularly shaped target volume.

Furthermore, our results suggest that carbon-ion beams may be more effective at countering radiation resistance due to tumor hypoxia. Generally, a bulky tumor has a larger hypoxic component (28), and Suzuki et al. reported that hypoxic cervical tumors exhibit a poorer LC rate with conventional radiotherapy (29). On the other hand, Nakano et al. reported favorable $\mathrm{LC}$ rates in both hypoxic and oxygenated tumors after C-ion RT (30). Thus, the therapeutic effect observed in bulky tumors in our study indicates that $\mathrm{C}$-ion $\mathrm{RT}$ is effective against the hypoxic component. Considering these observations, C-ion RT appears to be an effective treatment option for large, radioresistant tumors.

Distant metastases were frequently observed among our patients despite their favorable LC rates. Moreover, the DFS and OS rates were not too satisfactory when compared to CCRT with 3D IGBT (22-26). In the present study, patients had stage IIB-IVA diseases with large tumor sizes, and half of the patients had pelvic lymph metastases. However, these patients had not received chemotherapy for their initial treatment. To improve the DFS and OS rates, concurrent chemo-C-ion RT may be a worthwhile strategy for these patients.

Two patients developed grade $3 / 4$ rectal complications; the maximum doses to their GI tracts were 67.0 and $68.2 \mathrm{~Gy}$ (RBE), respectively. After starting protocol 9902, the dose to all GI tracts was strictly limited to $<60 \mathrm{~Gy}$ (RBE) using the revised treatment technique that involves 2-step cone-down for CTV. As a result, no grade 3 or higher complication was observed in protocol 9902 patients. Kato et al. reported the importance of dose constraints for the GI tract (17), and the present study further validates their notion with respect to long-term follow-up. Major clinical trials of CCRT using conventional brachytherapy for uterine cervical cancer showed that the incidence of grade 3 or higher rectal complications was $14-16 \%$ (5, 31). In comparison, 3D-IGBT has a markedly lower rate of severe late rectal complications; recent studies showed that the incidence of grade 3 or higher rectal complications using this modality was $0-7 \%$ (24-27). Although the number of patients in the present study was small, our results nevertheless illustrate that $\mathrm{C}$-ion RT for uterine cervical cancer with suitable dose constraints can reduce the risk of late rectal complications to levels observed with 3D-IGBT. With respect to late complications in the small intestine, no studies to date show long-term 3D-IGBT data. Nakano et al. reported that the rate of major complications in the small intestines at 20 years after conventional irradiation in patients with uterine cervical cancer can be as high as $8 \%$ (6). Although further follow-up is required, the low complication rate in the small intestines may be attributable to the excellent dose-sparing effect of C-ion RT.

\section{Conclusion}

We showed that C-ion RT produces favorable LC for locally advanced bulky cervical SqCC. In particular, C-ion RT with a higher dose is associated with excellent LC. Long-term safety was confirmed in the second (protocol 9902) study based on the establishment of dose constraints for the GI tract.

\section{Acknowledgements}

This work was supported by the Research Project with Heavy Ions at the National Institute of Radiological Sciences. The Authors thank all members of the Working Group of Gynecological Tumors for their generous support.

\section{References}

1 Mathew A and George PS: Trends in incidence and mortality rates of squamous cell carcinoma and adenocarcinoma of cervix - worldwide. Asian Pac J Cancer Prev 10(4): 645-650, 2009.

2 Rose PG, Bundy BN, Watkins EB, Thigpen JT, Deppe G, Maiman MA, Clarke-Pearson DL and Insalaco S: Concurrent cisplatin-based radiotherapy and chemotherapy for locally advanced cervical cancer. N Engl J Med 340(15): 1144-1153, 1999.

3 Whitney CW, Sause W, Bundy BN, Malfetano JH, Hannigan EV, Fowler WC Jr., Clarke-Pearson DL and Liao SY: Randomized comparison of fluorouracil plus cisplatin versus hydroxyurea as an adjuvant to radiation therapy in stages IIB-IVA carcinoma of the cervix with negative para-aortic lymph nodes: A Gynecologic Oncology Group and Southwest Oncology Group study. J Clin Oncol 17(5): 1339-1348, 1999.

4 Green JA, Kirwan JM, Tierney JF, Symonds P, Fresco L, Collingwood M and Williams CJ: Survival and recurrence after concomitant chemotherapy and radiotherapy for cancer of the uterine cervix: A systematic review and meta-analysis. Lancet 358(9284): 781-786, 2001. 
5 Eifel PJ, Winter K, Morris M, Levenback C, Grigsby PW, Cooper J, Rotman M, Gershenson D and Mutch DG: Pelvic irradiation with concurrent chemotherapy versus pelvic and paraaortic irradiation for high-risk cervical cancer: An update of Radiation Therapy Oncology Group Trial (RTOG) 90-01. J Clin Oncol 22(5): 872-880, 2004.

6 Nakano T, Kato S, Ohno T, Tsujii H, Sato S, Fukuhisa K and Arai T: Long-term results of high-dose-rate intracavitary brachytherapy for squamous cell carcinoma of the uterine cervix. Cancer 103(1): 92-101, 2005.

7 Perez CA, Grigsby PW, Chao KS, Mutch DG and Lockett MA: Tumor size, irradiation dose, and long-term outcome of carcinoma of uterine cervix. Int J Radiat Oncol Biol Phys 41(2): 307-317, 1998

8 Parker K, Gallop-Evans E, Hanna L and Adams M: Five years' experience treating locally advanced cervical cancer with concurrent chemoradiotherapy and high-dose-rate brachytherapy: results from a single institution. Int J Radiat Oncol Biol Phys 74(1): 140-146, 2009.

9 Syed AM, Puthawala AA, Abdelaziz NN, el-Naggar M, Disaia P, Berman M, Tewari KS, Sharma A, Londrc A, Juwadi S, Cherlow JM, Damore S and Chen YJ: Long-term results of low-dose-rate interstitial-intracavitary brachytherapy in the treatment of carcinoma of the cervix. Int J Radiat Oncol Biol Phys 54(1): 67-78, 2002.

10 Wakatsuki M, Ohno T, Yoshida D, Noda SE, Saitoh J, Shibuya K, Katoh H, Suzuki Y, Takahashi T and Nakano T: Intracavitary combined with CT-guided interstitial brachytherapy for locally advanced uterine cervical cancer: introduction of the technique and a case presentation. J Radiat Res 52(1): 54-58, 2011.

11 Tsujii $\mathrm{H}$ and Kamada T: A review of update clinical results of carbon ion radiotherapy. Jpn J Clin Oncol 42(8): 670-685, 2012.

12 Kanai T, Furusawa Y, Fukutsu K, Itsukaichi H, Eguchi-Kasai K and Ohara $\mathrm{H}$ : Irradiation of mixed beam and design of spreadout Bragg peak for heavy-ion radiotherapy. Radiat Res 147(1): 78-85, 1997

13 Kanai T, Endo M, Minohara S, Miyahara N, Koyama-ito H, Tomura H, Matsufuji N, Futami Y, Fukumura A, Hiraoka T, Furusawa Y, Ando K, Suzuki M, Soga F and Kawachi K: Biophysical characteristics of HIMAC clinical irradiation system for heavy-ion radiation therapy. Int J Radiat Oncol Biol Phys 44(1): 201-210, 1999.

14 Ando K, Koike S, Nojima K, Chen YJ, Ohira C, Ando S, Kobayashi N, Ohbuchi T, Shimizu W and Kanai T: Mouse skin reactions following fractionated irradiation with carbon ions. Int J Radiat Biol 74(1): 129-138, 1998.

15 Nakano T, Suzuki M, Abe A, Suzuki Y, Morita S, Mizoe J, Sato S, Miyamoto T, Kamada T, Kato $\mathrm{H}$ and Tsujii $\mathrm{H}$ : The phase I/II clinical study of carbon ion therapy for cancer of the uterine cervix. Cancer J Sci Am 5(6): 362-369, 1999.

16 Wakatsuki M, Kato S, Ohno T, Karasawa K, Ando K, Kiyohara $\mathrm{H}$, Tsujii H, Nakano T, Kamada T and Shozu M; Working Group of the Gynecological Tumor: Dose-escalation study of carbon ion radiotherapy for locally advanced squamous cell carcinoma of the uterine cervix (9902). Gynecol Oncol 132(1): 87-92, 2014.

17 Kato S, Ohno T, Tsujii H, Nakano T, Mizoe JE, Kamada T, Miyamoto $\mathrm{T}$, Tsuji $\mathrm{H}$, Kato $\mathrm{H}$, Yamada $\mathrm{S}$, Kandatsu $\mathrm{S}$, Yoshikawa K, Ezawa $\mathrm{H}$ and Suzuki M; Working Group of the Gynecological Tumor: Dose escalation study of carbon ion radiotherapy for locally advanced carcinoma of the uterine cervix. Int J Radiat Oncol Biol Phys 65(2): 388-397, 2006.
18 Wakatsuki M, Kato S, Kiyohara H, Ohno T, Karasawa K, Tamaki T, Ando K, Tsujii H, Nakano T, Kamada T, Shozu M and Working Group of the Gynecological Tumor: Clinical trial of prophylactic extended-field carbon-ion radiotherapy for locally advanced uterine cervical cancer (protocol 0508). PLoS One 10(5): e0127587, 2015.

19 Inaniwa T, Kanematsu N, Matsufuji N, Kanai T, Shirai T, Noda K, Tsuji H, Kamada T and Tsujii H: Reformulation of a clinicaldose system for carbon-ion radiotherapy treatment planning at the National Institute of Radiological Sciences, Japan. Phys Med Biol 60(8): 3271-3286, 2015

20 Cox JD, Stetz J and Pajak TF: Toxicity criteria of the Radiation Therapy Oncology Group (RTOG) and the European Organization for Research and Treatment of Cancer (EORTC). Int J Radiat Oncol Biol Phys 31(5): 1341-1346, 1995.

21 Toita T, Kitagawa R, Hamano T, Umayahara K, Hirashima Y, Aoki Y, Oguchi M, Mikami M and Takizawa K; Cervical Cancer (Vulva Cancer) Committee of Japanese Gynecologic Oncology Group (JGOG): Phase II study of concurrent chemoradiotherapy with high-dose-rate intracavitary brachytherapy in patients with locally advanced uterine cervical cancer: efficacy and toxicity of a low cumulative radiation dose schedule. Gynecol Oncol 126(2): 211-216, 2012.

22 Haie-Meder C, Pötter R, Van Limbergen E, Briot E, De Brabandere M, Dimopoulos J, Dumas I, Hellebust TP, Kirisits C, Lang S, Muschitz S, Nevinson J, Nulens A, Petrow P and Wachter-Gerstner N; Gynaecological (GYN) GEC-ESTRO Working Group: Recommendations from Gynaecological (GYN) GEC-ESTRO Working Group (I): concepts and terms in 3D image based 3D treatment planning in cervix cancer brachytherapy with emphasis on MRI assessment of GTV and CTV. Radiother Oncol 74(3): 235-245, 2005.

23 Pötter R, Haie-Meder C, Van Limbergen E, Barillot I, De Brabandere M, Dimopoulos J, Dumas I, Erickson B, Lang S, Nulens A, Petrow P, Rownd $J$ and Kirisits C; GEC ESTRO Working Group: Recommendations from gynaecological (GYN) GEC ESTRO working group (II): concepts and terms in 3D image-based treatment planning in cervix cancer brachytherapy 3D dose volume parameters and aspects of 3D image-based anatomy, radiation physics, radiobiology. Radiother Oncol 78(1): 67-77, 2006.

24 Ohno T, Noda SE, Okonogi N, Murata K, Shibuya K, Kiyohara H, Tamaki T, Ando K, Oike T, Ohkubo Y, Wakatsuki M, Saitoh JI and Nakano T: In-room computed tomography-based brachytherapy for uterine cervical cancer: results of a 5-year retrospective study. J Radiat Res 58(4): 543-551, 2017.

25. Ribeiro I, Janssen H, De Brabandere M, Nulens A, De Bal D, Vergote I and Van Limbergen E: Long term experience with 3D image guided brachytherapy and clinical outcome in cervical cancer patients. Radiother Oncol 120(3): 447-454, 2016.

26 Sturdza A, Pötter R, Fokdal LU, Haie-Meder C, Tan LT, Mazeron R, Petric P, Šegedin B, Jurgenliemk-Schulz IM, Nomden C, Gillham C, McArdle O, Van Limbergen E, Janssen H, Hoskin P, Lowe G, Tharavichitkul E, Villafranca E, Mahantshetty U, Georg P, Kirchheiner K, Kirisits C, Tanderup $\mathrm{K}$ and Lindegaard JC: Image guided brachytherapy in locally advanced cervical cancer: improved pelvic control and survival in RetroEMBRACE, a multicenter cohort study. Radiother Oncol 120(3): 428-433, 2016. 
27 Fokdal L, Sturdza A, Mazeron R, Haie-Meder C, Tan LT, Gillham C, Šegedin B, Jürgenliemk-Schultz I, Kirisits C, Hoskin P, Pötter R, Lindegaard JC and Tanderup K: Image guided adaptive brachytherapy with combined intracavitary and interstitial technique improves the therapeutic ratio in locally advanced cervical cancer: Analysis from the retroEMBRACE study. Radiother Oncol 120(3): 434-440, 2016.

28 Hall EJ and Giaccia AJ: Radiobiology for the radiologist. 7th ed. Philadelphia, Lippincott Williams \& Wilkins, 2012.

29 Suzuki Y, Nakano T, Ohno T, Kato S, Niibe Y, Morita S and Tsujii H: Oxygenated and reoxygenated tumors show better local control in radiation therapy for cervical cancer. Int $\mathrm{J}$ Gynecol Cancer 16(1): 306-311, 2006.

30 Nakano T, Suzuki Y, Ohno T, Kato S, Suzuki M, Morita S, Sato $\mathrm{S}$, Oka $\mathrm{K}$ and Tsujii $\mathrm{H}$ : Carbon beam therapy overcomes the radiation resistance of uterine cervical cancer originating from hypoxia. Clin Cancer Res 12(7): 2185-2190, 2006.
31 Whitney CW, Sause W, Bundy BN, Malfetano JH, Hannigan EV, Fowler WC Jr., Clarke-Pearson DL and Liao SY: Randomized comparison of fluorouracil plus cisplatin versus hydroxyurea as an adjunct to radiation therapy in stage IIB-IVA carcinoma of the cervix with negative para-aortic lymph nodes: A Gynecologic Oncology Group and Southwest Oncology Group study. J Clin Oncol 17(5): 1339-1348, 1999.
Received November 5, 2017

Revised November 14, 2017

Accepted November 15, 2017 